\section{OP0039 ALPN-303, AN ENHANCED, POTENT DUAL BAFFI APRIL ANTAGONIST ENGINEERED BY DIRECTED EVOLUTION FOR THE TREATMENT OF SYSTEMIC LUPUS ERYTHEMATOSUS (SLE) AND OTHER B CELL- RELATED AUTOIMMUNE DISEASES}

S. R. Dillon ${ }^{1}$, L. S. Evans ${ }^{2}$, K. E. Lewis ${ }^{1}$, J. Yang ${ }^{3}$, M. W. Rixon ${ }^{4}$, J. Kuijper ${ }^{5}$, D. Demonte ${ }^{5}$, J. Bhandari ${ }^{4}$, S. Levin ${ }^{2}$, K. Kleist ${ }^{1}$, S. Mudri ${ }^{1}$, S. Bort ${ }^{2}$,

D. Ardourel ${ }^{5}$, M. A. Seaberg ${ }^{6}$, R. Wang ${ }^{6}$, C. Gudgeon ${ }^{1}$, R. Sanderson ${ }^{6}$, M. F. Wolfson ${ }^{4}$, J. Hillson ${ }^{3}$, S. L. Peng ${ }^{3}{ }^{1}$ Alpine Immune Sciences, Translational Sciences, Seattle, United States of America; ${ }^{2}$ Alpine Immune Sciences Inc, Immunology, Seattle, United States of America; ${ }^{3}$ Alpine Immune Sciences Inc, Clinical Development, Seattle, United States of America; ${ }^{4}$ Alpine Immune Sciences Inc, Protein Therapeutics, Seattle, United States of America; ${ }^{5}$ Alpine Immune Sciences Inc, Protein Engineering, Seattle, United States of America; ${ }^{6}$ Alpine Immune Sciences Inc, Bioanalytical Sciences, Seattle, United States of America

Background: BAFF and APRIL are TNF superfamily members that form homoand heteromultimers that bind TACI and BCMA on B cells; BAFF also binds BAFF-R. BAFF and APRIL support B cell development, differentiation, and survival, particularly for plasmablasts and plasma cells, and play critical roles in the pathogenesis of $B$ cell-related autoimmune diseases. In nonclinical models, inhibition of either BAFF or APRIL alone mediates relatively modest effects, whereas their co-neutralization dramatically reduces $B$ cell function, including antibody production. Fc fusions of wild-type (WT) TACl (e.g. atacicept and telitacicept) target both BAFF and APRIL and have demonstrated promising clinical potential in e.g. systemic lupus erythematosus (SLE) and IgA nephropathy but have not yet clearly exhibited long-term and/or complete disease remissions.

Objectives: To generate a dual BAFF/APRIL antagonist with inhibitory activity superior to WT TACl and BCMA and with the potential to improve clinical outcomes in B cell-mediated diseases.

Methods: Our directed evolution platform was used to identify a potent variant TNFR domain (VTD) of TACl that exhibits significantly enhanced affinity for BAFF and APRIL as compared to WT TACl; this TACI VTD domain was fused to a human IgG Fc to generate the therapeutic candidate ALPN-303. ALPN-303 was evaluated for functional activity in: 1) human lymphocyte assays, 2) the NOD.Aec1Aec2 spontaneous model of Sjogren's syndrome (SjS), 3) the bm12-induced mouse model of lupus, 4 ) the (NZB/NZW)F $\mathrm{F}_{1}$ spontaneous model of lupus, and 5) preclinical rodent and cynomolgus monkey pharmacokinetic/pharmacodynamic studies.

Results: ALPN-303 inhibited BAFF- and APRIL-mediated signaling in vitro in human lymphocyte assays, with significantly lower $\mathrm{IC}_{50}$ values than WT TACI-Fc and belimumab comparators. In all mouse models evaluated, administration of ALPN-303 rapidly and significantly reduced key lymphocyte subsets including plasma cells, germinal center B cells, and follicular T helper cells. ALPN-303 significantly reduced autoantibodies and sialadenitis in the spontaneous SjS model, inhibited glomerular IgG deposition in the bm12-induced model of lupus, and potently suppressed anti-dsDNA autoAbs, blood urea nitrogen levels, proteinuria, sialadenitis, kidney lesions, and renal immune complex deposition in the NZB/W lupus model. As compared to WT TACI-Fc, ALPN-303 exhibited higher serum exposure and significantly and persistently decreased titers of serum $\operatorname{lgM}, \lg G$, and IgA antibodies in mice and cynomolgus monkeys (Figure 1).

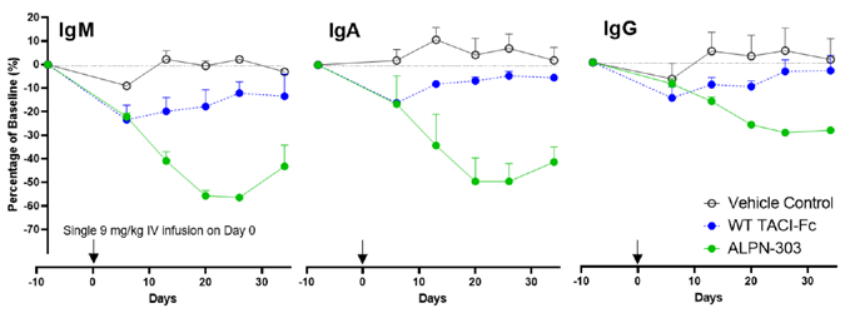

Figure 1. ALPN-303 induces more potent suppression, as compared to WT TACI-Fc, of serum immunoglobulins following a single $9 \mathrm{mg} / \mathrm{kg}$ IV infusion (on Day 0; arrows) in female cynomoIgus monkeys.

Conclusion: ALPN-303 is a potent BAFF/APRIL antagonist derived from our directed evolution platform that consistently demonstrates encouraging immunomodulatory activity and efficacy in vitro and in vivo, superior in preclinical studies to anti-BAFF antibody and WT TACI-Fc. This novel Fc fusion molecule demonstrates favorable preliminary developability characteristics, including higher serum exposures and more potent immunosuppressive activities, which may enable lower clinical doses and/or longer dosing intervals than WT TACI-Fc therapeutics. ALPN303 may thus be an attractive development candidate for the treatment of multiple autoimmune and inflammatory diseases, particularly B cell-related diseases such as SLE, SjS, and other connective tissue diseases. Preclinical development is underway to enable the initiation of clinical trials later this year.
Disclosure of Interests: Stacey R. Dillon Shareholder of: Alpine Immune Sciences, Bristol Myers Squibb, Employee of: Alpine Immune Sciences, Bristol Myers Squibb, Lawrence S. Evans Shareholder of: Alpine Immune Sciences Employee of: Alpine Immune Sciences, Katherine E. Lewis Shareholder of: Alpine Immune Sciences, Employee of: Alpine Immune Sciences, Jing Yang Shareholder of: Alpine Immune Sciences, Employee of: Alpine Immune Sciences, Mark W. Rixon Shareholder of: Alpine Immune Sciences, Employee of: Alpine Immune Sciences, Joe Kuijper Shareholder of: Alpine Immune Sciences, Employee of: Alpine Immune Sciences, Dan Demonte Shareholder of: Alpine Immune Sciences, Employee of: Alpine Immune Sciences, Janhavi Bhandari Shareholder of: Alpine Immune Sciences, Employee of: Alpine Immune Sciences, Steve Levin Shareholder of: Alpine Immune Sciences, Employee of: Alpine Immune Sciences, Kayla Kleist Shareholder of: Alpine Immune Sciences, Employee of: Alpine Immune Sciences, Sherri Mudri Shareholder of: Alpine Immune Sciences, Employee of: Alpine Immune Sciences, Susan Bort Shareholder of: Alpine Immune Sciences, Employee of: Alpine Immune Sciences, Daniel Ardourel Shareholder of: Alpine Immune Sciences, Employee of: Alpine Immune Sciences, Michelle A. Seaberg Shareholder of: Alpine Immune Sciences, Employee of: Alpine Immune Sciences, Rachel Wang Shareholde of: Alpine Immune Sciences, Employee of: Alpine Immune Sciences, Chelsea Gudgeon Shareholder of: Alpine Immune Sciences, Employee of: Alpine Immune Sciences, Russell Sanderson Shareholder of: Alpine Immune Sciences, Employee of: Alpine Immune Sciences, Martin F. Wolfson Shareholder of: Alpine Immune Sciences, Employee of: Alpine Immune Sciences, Jan Hillson Shareholder of: Alpine Immune Sciences, Employee of: Alpine Immune Sciences, Stanford L. Peng Shareholder of: Alpine Immune Sciences, Employee of: Alpine Immune Sciences

DOI: 10.1136/annrheumdis-2021-eular.727

\section{OP0040 HIPPOCAMPAL IMMUNE CELL TRAFFICKING AND A MYELOID PREDOMINANT INFLAMMATORY RESPONSE WITH ENHANCED ANTIGEN PRESENTATION AND DECREASED LEVELS OF NEUROTRANSMITTERS UNDERLY THE NEUROPSYCHIATRIC PHENOTYPE OF THE NZW/NZB MURINE LUPUS MODEL}

D. Nikolopoulos ${ }^{1,2}$, T. Manolakou ${ }^{1}$, A. Filia ${ }^{1}$, M. Nakos-Bimpos ${ }^{1}$, A. Polissidis ${ }^{1}$, D. Boumpas ${ }^{1,2} .{ }^{1}$ Biomedical Research Foundation Academy of Athens, Center of Translational Research, Athens, Greece; ${ }^{2}$ National and Kapodistrian University of Athens, Rheumatology and Clinical Immunology Unit, 4th Department of Medicine, Athens, Greece

Background: Neuropsychiatric events are common in patients with systemic lupus erythematosus (SLE), yet the underlying pathogenesis remains ill-defined, as the access to brain tissue is limited. We have previously shown that NZW/ NZB F1 murine lupus model recapitulates the neuropsychiatric lupus phenotype including depressive-like behavior, increased rates of anxiety, cognitive dysfunction and motor disturbances, both at pre-nephritic and nephritic stages of the disease.

Objectives: To dissect specific regions in the brain, which account for this phenotype and elucidate inflammatory and non-inflammatory mechanisms involved. Methods: Four distinct brain regions (hippocampus, amygdala, striatum and pre-frontal cortex) were dissected from brains of female C57BL/6 (WT) and NZW/NZB F1 mice at the age of 3 months (pre-nephritic) and 6 months (nephritic stage) ( $n=5-8 /$ condition/experiment). Since most of the behavioral phenotype corresponds to the hippocampus, we first examined in depth the hippocampal pathology by bulk RNA sequencing, measurements of neurotransmitters levels via high-performance liquid chromatography (HPLC) and by immunophenotyping via flow cytometry analyses. For comparisons, statistical significance was indicated as a two-sided $\mathrm{P}<0.05$

Results: Transcriptomic analysis revealed aberrant immune mediated response in the hippocampus of 6 month-old lupus mice compared to WT. Specifically, inflammatory pathways including both innate and adaptive immune responses, increased cytokine production, increased antigen presentation and immune cell trafficking, along with increased apoptosis and decreased cell proliferation suggest that immune aberrancies may lead to neuronal damage. These aberrancies were present in mice at 3 month-old, yet were progressed with time being more prominent at 6 month of age in lupus hippocampus. The RNA sequencing date were validated by immunophenotyping on lupus hippocampus demonstrating increased reactive GFAP+ astrocytes both at 3 and 6-month old mice. Activated IBA1+ microglia and CD11b+CD45hi CNS myeloid cells were increased only at 6 months of age. Furthermore, increased immune cell infiltration from the periphery including lymphocytes (CD45+CD11b-) mainly $T$ cells (CD4+/CD8+) and monocytes (CD45+CD11b+Ly6G-Ly6C+), was evident only in 6 month-old lupus hippocampus compared to WT. Importantly, microglia cells in lupus hippocampus at 6 but not at 3 month of age, exhibited increased expression of antigen presenting markers including CD80, CD86 and MHC-I 